Pacific Journal of Mathematics

THE CLOSED RANGE MODULUS OF OPERATORS 


\title{
THE CLOSED RANGE MODULUS OF OPERATORS
}

\author{
F.-H. VASILESCU
}

In order to describe a certain "lattice type" behavior of the closed subspaces in Banach spaces, with regard to the action of linear operators, we introduce the notion of closed range modulus. Some consequences for the spectral theory of commuting finite systems of linear operators are then obtained.

1. Let $X$ and $Y$ be two complex Banach spaces and $\mathfrak{C}(X, Y)$ $(\mathfrak{B}(X, Y))$ the family of all closed (bounded) linear operators from $X$ to $Y$. For any $T \in \mathfrak{S}(X, Y)$ denote by $\mathfrak{D}(T)$ the domain of $T$ and by $\Re(T)$ the range of $T$. If $\mathrm{Z} \subset X$ is any closed subspace then $T \mid Z$ means the restriction of $T$ on $Z$, i.e. the operator defined on $\mathfrak{D}(T \mid Z)=\mathfrak{D}(T) \cap Z$ as $T$. Denote also by $\Re(T)$ the null-space of $T$ and by $\Re_{T}(X, Y)$ the family of all closed subspaces $Z$ of $X$ with the property that $\Re(T \mid Z)$ is a closed subspace of $Y$. Since $\mathfrak{R}(T \mid Z)$ is always closed, then for any $Z \in \mathfrak{R}_{T}(X, Y)$ the operator

$$
\tau_{Z}: T x \in \mathfrak{R}(T \mid Z) \longrightarrow x+\mathfrak{R}(T \mid Z) \in X / \mathfrak{N}(T \mid Z)
$$

is everywhere defined, hence bounded by the closed graph theorem. Denote by $\left\|\tau_{z}\right\|$ its norm and for any $\mathfrak{R} \subset \Re_{T}(X, Y)$ let us define

$$
\kappa(T, \Re)=\sup _{Z \in \Re}\left\|\tau_{Z}\right\| \text {. }
$$

When $\Re=\Re_{T}(X, Y)$ we put simply $\kappa(T)=\kappa(T, \Re)$.

Then number $\kappa(T)(\kappa(T, \Re)$ ) will be called the closed range modulus of $T$ (on $\mathfrak{R}$ ). It is clear that $0 \leqq \kappa(T, \mathfrak{R}) \leqq+\infty$ and $\kappa(T, \mathfrak{R})=0$ if and only if $T \mid Z=0$, for any $Z \in \Re$.

Let us remark that the case $\Re=\{Z\}$ has been considered in [8, IV. 5.1].

There are simple examples which show that $\kappa(T, \Re)$ may be infinite. However, the class of operators having a finite closed range modulus is reasonably large, as shown by the following result.

Proposition 1.1. If $T \in \mathbb{E}(X, Y)$ has a bounded inverse then $\kappa(T)=\left\|T^{-1}\right\|$.

Proof. Take $Z \in \mathfrak{R}_{T}(X, Y)$. Then the operator $\tau_{Z}$, given by (1.1), has the form $\tau_{Z}: T x \rightarrow x$, for every $x \in \mathfrak{D}(T \mid Z)$. Therefore we can write

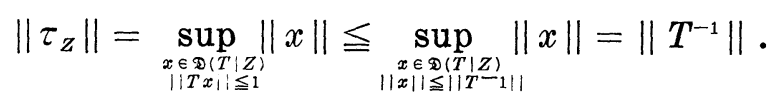


When $Z=X$ we have $\tau_{z}=T^{-1}$, hence $\kappa(T)=\left\|T^{-1}\right\|$.

2. In this section we shall obtain some "lattice type" consequences of the finiteness of the closed range modulus.

Lemma 2.1. Suppose that $\Re \subset \Re_{T}(X, Y)$ and $\kappa(T, \Re)<+\infty$. If $M>\kappa(T, \Re)$ is a fixed constant, then for any $Z \in \Re$ and for any $y \in \Re(T \mid Z)$ there is an $x \in \mathfrak{D}(T \mid Z)$ such that $T x=y$ and $\|x\| \leqq$ $M\|y\|$.

Proof. The assertion is a simple consequence of the open map principle.

TheOREM 2.2. Let $T$ be in $\mathfrak{S}(X, Y)$ and $\Re=\left\{Z_{\alpha}\right\}_{\alpha}$ an increasingly directed family in $\Re_{T}(X, Y)$. Suppose that $\kappa(T, \Re)<\infty$ and $\overline{\Re(T \mid Z)}=$ c.l.m. $\left\{\Re\left(T \mid Z_{\alpha}\right)\right\}_{\alpha}$, where $Z=$ c.l.m. $\left\{Z_{\alpha}\right\}_{\alpha}$. Then $Z \in$ $\Re_{T}(X, Y)$.

Proof. Let $y$ be in $\overline{\Re(T \mid Z)}$ arbitrary. Then there is a sequence $y_{n} \in \Re\left(T \mid Z_{\alpha_{n}}\right)$ such that $y_{n} \rightarrow y$ as $n \rightarrow \infty$. We shall use an approximation procedure, inspired by the proof of the closed graph theorem [8]. With no loss of generality we may suppose that $\sum_{n=1}^{\infty} \| y_{n+1}-$ $y_{n} \|<\infty$. Fix a constant $M>\kappa(T, \Re)$. We shall apply succesively Lemma 2.1. Choose $x_{1} \in \mathfrak{D}\left(T \mid Z_{\alpha_{1}}\right)$ such that $T x_{1}=y_{1}$ and $\left\|x_{1}\right\| \leqq$ $M\left\|y_{1}\right\|$. Then, for an arbitrary $n \geqq 1$, we may find an index $\beta_{n}$ such that $Z_{\beta_{n}} \supset Z_{\alpha_{n}}+Z_{\alpha_{n+1}}$ and an element $x_{n+1} \in Z_{\beta_{n}}$ with the properties $T x_{n+1}=y_{n+1}-y_{n}$ and $\left\|x_{n+1}\right\| \leqq M\left\|y_{n+1}-y_{n}\right\|$. According to our choice of $x_{n}$ we have

$$
\sum_{n=1}^{\infty}\left\|x_{n}\right\| \leqq M\left(\left\|y_{1}\right\|+\sum_{n=1}^{\infty}\left\|y_{n+1}-y_{n}\right\|\right),
$$

hence $x=\sum_{n=1}^{\infty} x_{n} \in X$. Moreover, as $T$ is a closed operator and $T\left(\sum_{n=1}^{k} x_{n}\right)=y_{k}$ we infer that $x \in \mathfrak{D}(T)$ and $T x=y$.

CoRollary 2.3. Suppose that $T \in \mathfrak{B}(X, Y)$ and let $\Re=\left\{Z_{\alpha}\right\}_{\alpha}$ be an increasingly directed family in $\Re_{T}(X, Y)$. If $\kappa(T, \Re)<+\infty$ then $Z \in \mathfrak{R}_{T}(X, Y)$, where $Z=$ c.l.m. $\left\{Z_{\alpha}\right\}_{\alpha}$.

Proof. We have only to notice that the assumption $T \in \mathfrak{B}(X, Y)$ implies $\overline{\Re(T \mid Z)}=$ c.l.m. $\left\{\Re\left(T \mid Z_{\alpha}\right)\right\}_{\alpha}$.

TheOREM 2.4. Suppose that $X, Y$ are the duals of the Banach spaces $X_{*}, Y_{*}$ respectively, and $T \in \mathfrak{C}(X, Y)$ is the adjoint of a densely defined operator $T_{*} \in \mathfrak{S}\left(Y_{*}, X_{*}\right)$. If $\left\{Z_{\alpha}\right\}_{\alpha}$ is a decreasingly directed 
family of $w^{*}$-closed subspaces in $\Re_{T}(X, Y)$ and $\kappa(T, \Re)<\infty$ then $Z=\bigcap_{\kappa} Z_{\alpha}$ belongs to $\Re_{T}(X, Y)$.

Proof. Assume that $y \in \overline{\Re(T \mid Z)}$, hence $y=\lim _{n} y_{n}$, with $y_{n} \in$ $\Re(T \mid Z)$, for any natural $n$. We may suppose that $\sum_{n=1}^{\infty} \| y_{n+1}-$ $y_{n} \|<+\infty$ and proceed as in the proof of Theorem 2.2. Namely, for any $\alpha$ we can choose a sequence $x_{n, \alpha} \in \mathfrak{D}\left(T \mid Z_{\alpha}\right)$ such that $T x_{1, \alpha}=y_{1}$, $T x_{n+1, a}=y_{n+1}-y_{n}(n \geqq 1),\left\|x_{1, \alpha}\right\| \leqq M\left\|y_{1}\right\|$ and $\left\|x_{n+1, \alpha}\right\| \leqq M \| y_{n+1}-$ $y_{n} \|(n \geqq 1)$. Consequently, $x_{\alpha}+\sum_{n=1}^{\infty} x_{n, \alpha} \in Z_{\alpha}$ Since $T$ is closed, we infer $x_{\alpha} \in \mathfrak{D}\left(T \mid Z_{\alpha}\right)$ and $T x_{\alpha}=y$. Moreover,

$$
\left\|x_{\alpha}\right\| \leqq M\left(\left\|y_{1}\right\|+\sum_{n=1}^{\infty}\left\|y_{n+1}-y_{n}\right\|,\right.
$$

therefore $\left\{x_{\alpha}\right\}_{\alpha}$ is uniformly bounded. Let $x$ be a cluster point of $\left\{x_{\alpha}\right\}_{\alpha}$ in the $w^{*}$-topology of $X$. Since $\left\{Z_{\alpha}\right\}_{\alpha}$ is a decreasingly directed family of $w^{*}$-closed subspaces, we get $x \in Z$. Let us show that $x \in \mathfrak{D}(T)$ and $T x=y$. Indeed, if $\varepsilon>0$ and $f \in \mathfrak{D}\left(T_{*}\right)$ are arbitrary, there is an index $\alpha$ such that

$$
\left|x\left(T_{*} f\right)-x_{\alpha}\left(T_{*} f\right)\right|<\varepsilon .
$$

Since $T x_{\alpha}=y$ and $\varepsilon>0$ is arbitrary, we obtain $x\left(T_{*} f\right)=y(f)$ for any $f \in \mathfrak{D}\left(T_{*}\right)$, whence $x \in \mathfrak{D}(T)$ and $T x=y$.

Corollary 2.5. Suppose that $X, Y$ are reflexive Banach spaces and let $T$ be a densely defined operator in $\mathfrak{S}(X, Y)$. If $\Re=\left\{Z_{\alpha}\right\}_{\alpha}$ is a decreasingly directed family in $\mathfrak{R}_{T}(X, Y)$ such that $\kappa(T, \Re)<\infty$ then $Z=\bigcap_{\alpha} Z_{\alpha}$ belongs to $\Re_{T}(X, Y)$.

Proof. The result follows from the previous theorem, since $T=T^{* *}$ and the $w^{*}$-topology of $X$ coincides with its $w$-topology.

3. From now on we restrict ourselves to the case $X=Y$ and consider only bounded operators. We shall put $\mathfrak{B}(X)$ instead of $\mathfrak{B}(X, X)$.

For the sake of simplicity, an operator $T \in \mathfrak{B}(X)$ with the property $T^{2}=0$ will be called a 2-nilpotent. These operators are related to the definition of the joint spectrum of a commuting system of linear operators, as described in the next section.

A 2-nilpotent operator $T \in \mathfrak{B}(X)$ will be called exact (on $X$ ) if $\mathfrak{R}(T)=\mathfrak{R}(T)$.

THEOREM 3.1. Let $T$ be a 2-nilpotent operator on $X$ and $\mathfrak{R}=\left\{Z_{\alpha}\right\}_{\alpha}$ 
an increasingly directed family of closed subspaces of $X$, invariant under $T$. If $T \mid Z$ is exact for any $\alpha$ and $\kappa(T, \Re)<\infty$ then $T \mid Z$ is exact, where $Z=$ c.l.m. $\left\{Z_{\alpha}\right\}$.

Proof. Consider $y \in \mathfrak{R}(T \mid Z)$. Then there is a sequence $y_{n} \in Z_{\alpha_{n}}$ such that $y=\lim _{n} y_{n}$. With no loss of generality we may suppose that $T y_{n}=0$ for any $n$. Indeed, if $y_{n}^{\prime} \rightarrow y, y_{n}^{\prime} \in Z_{\alpha_{n}}$, then $T y_{n}^{\prime} \rightarrow$ $T y=0$, therefore if $M>\kappa(T, \Re)$ is fixed, we may choose $v_{n} \in Z_{\alpha_{n}}$ such that $\left\|v_{n}\right\| \leqq M\left\|T y_{n}\right\|$ and $T v_{n}=T y_{n}^{\prime}$. In particular, $v_{n} \rightarrow 0$ as $n \rightarrow \infty$. If we put $y_{n}=y_{n}^{\prime}-v_{n}$, we have $T y_{n}=0$ and $y_{n} \rightarrow y$ as $n \rightarrow \infty$.

We can proceed now as in the proof of Theorem 2.2. Namely, assuming $\sum_{n=1}^{\infty}\left\|y_{n+1}-y_{n}\right\|<\infty$ we can find a sequence $\left\{x_{n}\right\}$ in $Z$ such that $T x_{1}=y_{1}, T x_{n+1}=y_{n+1}-y_{n}$ and the series $x=\sum_{n=1}^{\infty} x_{n}$ is convergent in $Z$. Then $T x=y$, hence $T \mid Z$ is exact.

Theorem 3.2. Assume that $X$ is the dual of the Banach space $X_{*}$ and $T$ is the adjoint of $T_{*} \in \mathfrak{B}\left(X_{*}\right)$. Let $\mathfrak{R}=\left\{Z_{\alpha}\right\}_{\alpha}$ be a decreasingly directed family of $w^{*}$-closed subspaces of $X$, invariant under $T$. If $T \mid Z$ is exact for any $\alpha$ and $\kappa(T, \Re)<\infty$ then $T \mid Z$ is exact, where $Z=\bigcap_{\alpha} Z_{\alpha}$.

Proof. Let $y$ be in $\Re(T \mid Z)$, therefore for any $\alpha, y=T x_{\alpha}$ with $x_{\alpha} \in Z_{\alpha}$. Since $\left\{x_{\alpha}\right\}$ can be chosen uniformly bounded, we may find, as in the proof of Theorem 2.4, a vector $x \in Z$ such that $y=T x$.

4. First of all we recall the definition of the joint spectrum of a commuting system $T=\left(T_{1}, \cdots, T_{n}\right) \subset \mathfrak{B}(X)$, in the sense of Taylor's [9] (see also [12]).

Denote by $\Lambda^{p}(n, X)$ the set of all antisymmetric functions defined on $\{1, \cdots, n\}^{p}$, with values in $X$, for any natural $p$. Let us denote by $\delta_{T}^{p}: \Lambda^{p}(n, X) \rightarrow \Lambda^{p+1}(n, X)$, the operator defined as

$$
\delta_{T}^{p} \xi\left(\nu_{1}, \cdots, \nu_{p+1}\right)=\sum_{j=1}^{p+1}(-1)^{j+1} T \nu_{j} \xi\left(v_{1}, \cdots, \hat{\nu}_{j} \cdots, \nu_{p+1}\right),
$$

where the symbol " $\wedge$ " means that the corresponding index is omitted. One can easily prove that $\delta_{T}^{p+1} \delta_{T}^{p}=0$. We define also $\Lambda^{0}(n, X)=X$ and $\delta_{T}^{0} x(\nu)=T_{\nu} x$, for any $x \in X$ and $\nu=1, \cdots, n$.

The system $T$ is called nonsingular (singular) if $\Re\left(\delta_{T}^{p}\right)=\mathfrak{R}\left(\delta_{T}^{p+1}\right)$ for every nonnegative integer $p$ (there is a $p$ such that $\Re\left(\delta_{T}^{p}\right) \neq$ $\left.\mathfrak{N}\left(\delta_{T}^{p+1}\right)\right)$.

The spectrum of $T$ on $X$, denoted by $\delta(T, X)$, is defined as the set of all points $z=\left(z_{1}, \cdots, z_{n}\right) \in C^{n}$ such that $z-T=\left(z_{1}-T_{1}, \cdots\right.$, $\left.z_{n}-T_{n}\right)$ is singular on $X$. 
These things can be considered in a slightly different manner. Namely, it is clear that we can identify $\Lambda^{p}(n, X)(1 \leqq p \leqq n)$ with the direct sum

$$
\bigoplus_{1 \leqq \nu_{1}<\cdots<\nu_{p} \leqq n} X
$$

Let us set then

$$
X^{(n)}=\bigoplus_{p=0}^{n} \Lambda^{p}(n, X)
$$

and

$$
\delta_{T}=\bigoplus_{p=0}^{n} \delta_{T}^{p}
$$

The space $X^{(n)}$ is a direct sum of $2^{n}$ copies of $X$ and $\delta_{T}$ is 2-nilpotent on $X^{(n)}$ (provided that $\delta_{T}^{n}=0: \Lambda^{n}(n, X) \rightarrow \Lambda^{0}(n, X)$ ). Since $\Lambda^{p}(n, X)$ is null for $p>n$, therefore it has no contribution in the definition of the spectrum it is easy to see that a system $T=\left(T_{1}\right.$, $\left.\cdots, T_{n}\right)$ is nonsingular on $X$ if and only if the 2-nilpotent operator $\delta_{T}$ is exact on $X^{(n)}$.

The definition of the joint spectrum allows to recapture many spectral properties of the one-dimensional case, including the functional calculus with analytic functions [9], [10]. However, there are some simple properties which have not direct variants in several dimensions. One of them is given by the next result.

Proposition 4.1. Suppose that $T \in \mathfrak{B}(X)$ is invertible and let $\left\{Z_{\alpha}\right\}_{\alpha}$ be an increasingly directed family of closed subspaces of $X$, invariant under $T$, such that $T \mid Z_{\alpha}$ is invertible for any $\alpha$. Then $T \mid Z$ is invertible, where $Z=$ c.l.m. $\left\{Z_{\alpha}\right\}_{\alpha}$.

Proof. It is sufficient to show that $T \mid Z$ is surjective. Indeed, if $y \in Z$ then $y=\lim _{n} y_{n}$, where $y_{n} \in Z_{\alpha_{n}}$, therefore $y_{n}=T x_{n}$, with $x_{n} \in Z_{\alpha_{n}}$. Moreover,

$$
\lim _{n \rightarrow \infty} x_{n}=\lim _{n \rightarrow \infty}\left(T \mid Z_{\alpha_{n}}\right)^{-1} y_{n}=\lim _{n \rightarrow \infty} T^{-1} y_{n},
$$

consequently $x=\lim _{n} x_{n} \in Z$ and $T x=y$.

A direct version of Proposition 4.1 is not possible for more than one dimension.

ExAmple. Let $Z$ be a separable Hilbert space and set $Z_{k}=Z$ for $k=1,2,3, \cdots$. Suppose that every $Z_{k}$ has an orthonormal basis of the form $\left\{e_{j}^{k}\right\}_{j=\rightarrow \infty}^{+\infty}$. Define $X=\bigoplus_{k=1}^{\infty} Z_{k}$. Let $T_{k}$ be the bilateral translation on $Z_{k}$, namely 


$$
T_{k} e_{j}^{k}=e_{j+1}^{k}, \quad(j \in Z)
$$

and denote by $T$ the direct sum $\bigoplus_{k+1}^{\infty} T_{k}$. Let now $\left\{\lambda_{k}\right\}_{k=1}^{\infty}$ a sequence of complex numbers, $\lambda_{k} \neq 0$ for any $k, \lambda_{k} \rightarrow 0$ as $k \rightarrow \infty$. We define on every $Z_{k}$ the operator $S_{k}=\lambda_{k}$ and let us put $S=\bigoplus_{k=1}^{\infty} S_{k}$. It is obvious that $T S=S T$. Since the operator $T$ is unitary on $X$, thus invertible, it follows that the system $(T, S)$ is nonsingular on $X$ ([9]; see also the proof of Theorem 4.5).

Consider now the subspaces

$$
Z_{k}^{+}=\text {c.l.m. }\left\{e_{j}^{k}\right\}_{j=0}^{\infty}, \quad k=1,2, \cdots
$$

and define $X_{n}^{+}=\bigoplus_{k=1}^{n} Z_{k}^{+}, X^{+}=\bigoplus_{k=1}^{\infty} Z_{k}$. It is clear that the spaces $X_{n}^{+}$and $X^{+}$are invariant under $T$ and $S$, for any $n$. Furthermore, the system $\left(T\left|X_{n}^{+}, S\right| X_{n}^{+}\right)$is nonsingular on $X_{n}^{+}$since the operator $S \mid X_{n}^{+}$is invertible (because of the choice of the numbers $\lambda_{k}, 1 \leqq k \leqq n$ ). On the other hand, $\left(T\left|X^{+}, S\right| X^{+}\right)$is singular. Indeed, it is sufficient to show that $X^{+} \neq T X^{+}+S X^{+}$. Let $\xi$ be in $X^{+}$of the form $\xi=\sum_{k=1}^{\infty} \xi_{k} e_{0}^{k}$. If $\xi=T \zeta+S \eta$, we would have, for the coefficients $\eta_{k}$ of $\eta$ corresponding to $e_{0}^{k}$, the relations

$$
\lambda_{k} \eta_{k}=\xi_{k}, \quad k=1,2,3, \cdots
$$

which is not possible for any choice of $\xi$ of the given form, because $\lambda_{k} \rightarrow 0$ as $k \rightarrow \infty$. In this way we have shown that the nonsingularity of $(T, S)$ on $X$ and on any $X_{n}^{+}$does not imply the nonsingularity of $(T, S)$ on $X^{+}=$c.l.m. $\left\{X_{n}^{+}\right\}_{n}$.

There is a result which is "symmmetrical" to Proposition 4.1.

Proposition 4.2. Suppose that $T \in \mathfrak{B}(X)$ is invertible and let $\left\{Z_{\alpha}\right\}_{\alpha}$ be a decreasingly directed family of closed subspaces of $X$, invariant under $T$, such that $T \mid Z_{\alpha}$ is invertible for each $\alpha$. Then $T \mid Z$ is invertible, where $Z=\bigcap_{\alpha} Z_{\alpha}$.

Proof. It is easy to see that $T \mid Z$ is injective and surjective.

According to expectation, Proposition 4.2 does not have a direct variant in several dimensions. An example in this sense can be easily obtained from the above Example. Indeed, with the same notations, consider the system $\left(T^{*}, S^{*}\right)$ and the family of subspaces $\left\{\left(X_{n}^{+}\right)^{\perp}\right\}_{n=1}^{\infty}$. Since the nonsingularity is preserved by the duality and the space $\left(X_{n}^{+}\right)^{\perp}$ is isomorphic to the dual space $\left(X / X_{n}\right)^{*}$, we obtain that $\left(T^{*}, S^{*}\right)$ is nonsingular on $\left(X_{n}^{+}\right)^{\perp}$ because $(T, S)$ is nonsingular on both $X$ and $X_{n}^{+}$(see [9, Lemma 1.2]). Analogously, $\left(T^{*}, S^{*}\right.$ ) cannot be nonsingular on $\bigcap_{n}\left(X_{n}^{+}\right)^{\perp}=\left(X^{+}\right)^{\perp}$ since $(T, S)$ is singular on $X^{+}$.

In spite of these rather disappointing examples, there are cases 
when both Propositions 4.1 and 4.2 have extensions in several variables. These cases are consequences of the finiteness of a certain closed range modulus.

Consider again a system $T=\left(T_{1}, \cdots, T_{n}\right)$ of commuting operators on the Banach space $X$. If $Z \subset X$ is a closed subspace invariant under $T$ (i.e. invariant under $T_{j}$ for $j=1, \cdots, n$ ), define $Z^{(n)}$ by the formula (4.1) and notice that $Z^{(n)}$ is a subspace of $X^{(n)}$, invariant under $\hat{o}_{T}$. If $\Re=\left\{Z_{\alpha}\right\}_{\alpha}$ is a family of such subspaces, then denote by $\Re^{(n)}$ the corresponding family $\left\{Z_{\alpha}^{(n)}\right\}_{\alpha}$. We define also the closed range modulus of $T$ on $\Re$ by the formula

$$
\kappa(T, \Re)=\kappa\left(\delta_{T}, \Re^{(n)}\right) .
$$

For any subspace $Z \subset X$ invariant under $T=\left(T_{1}, \cdots, T_{n}\right)$, the notation $T \mid Z$ stands for the system $\left(T_{1}\left|Z, \cdots, T_{n}\right| Z\right)$.

THEOREM 4.3. Let $T=\left(T_{1}, \cdots, T_{n}\right)$ be a commuting system of operators in $\mathfrak{B}(X)$ and $\Re=\left\{Z_{\alpha}\right\}_{\alpha}$ an increasingly directed family of closed subspaces of $X$, invariant under $T$. If $T \mid Z_{\alpha}$ in nonsingular for any $\alpha$ and $\kappa(T, \Re)<\infty$ then $T \mid Z$ is nonsingular, where $Z=$ c.l.m. $\left\{Z_{\alpha}\right\}_{\alpha}$.

Proof. Since $Z^{(n)}=$ c.l.m. $\left\{Z_{\alpha}^{(n)}\right\}_{\alpha}$, this theorem is Theorem 3.1 rewritten.

Analogously, we get from Theorem 3.2 the following

THEOREM 4.4. Assume that $X$ is the dual of $X_{*}$ and $T=$ $\left(T_{1}, \cdots, T_{n}\right)$ is the adjoint of the system $T_{*}=\left(T_{1^{*}}, \cdots, T_{n^{*}}\right), T_{*}$ acting in $X_{*}$. If $\Re=\left\{Z_{\alpha}\right\}_{\alpha}$ is a decreasingly directed family of $w^{*}$-closed subspaces of $X$, invariant under $T$, such that $T \mid Z_{\alpha}$ is nonsingular for any $\alpha$ and $\kappa(T, \Re)<\infty$, then $T \mid Z$ is nonsingular, where $Z=\bigcap_{\alpha} Z_{\alpha}$.

We shall end this section with a result of finiteness for the closed range modulus of a commuting system of operators, in a special case.

THEOREM 4.5. Let $T=\left(T_{1}, \cdots, T_{n}\right)$ be a commuting system of operators on $X$ and assume that $T_{1} V_{1}+\cdots+T_{n} V_{n}=1$, where $V_{1}, \cdots, V_{n}$ are operators in the commutant of $T$ in $\mathfrak{B}(X)$. If $\mathfrak{R}$ is any family of closed subspaces of $X$, invariant for the action of $T_{j}$ and $V_{j}(j=1, \cdots, n)$, then $\kappa(T, \Re)<\infty$.

Proof. We have to show that the maps 


$$
\Re\left(\delta_{T \mid Z}\right) \longrightarrow Z^{(n)} / \mathfrak{R}\left(\delta_{T \mid Z}\right),
$$

defined as in (1.1), are uniformly bounded for $Z \in \Re$. We shall use an idea from [9, Lemma 1.1]. Fix $Z \in \Re$ and take $\xi^{p} \in \Lambda^{p}(n, Z)$ with the property $\delta_{T}^{p} \xi^{p}=0$. Let us define the element $\eta^{p} \in \Lambda^{p-1}(n, Z)$ by the formula

$$
\eta^{p}\left(\nu_{1}, \cdots, \nu_{p_{-1}}\right)=\sum_{j=1}^{n} V_{j} \xi\left(j, \nu_{1}, \cdots, \nu_{p-1}\right) .
$$

Then we can write

$$
\begin{aligned}
\delta_{T}^{p-1} \eta^{p}\left(\nu_{1}, \cdots, \nu_{p}\right) & =\sum_{j=1}^{p}(-1)^{j+1} T_{\nu_{j}} \sum_{k=1}^{n} V_{k} \xi^{p}\left(k, \nu_{1}, \cdots, \hat{\nu}_{j}, \cdots, \nu_{p}\right) \\
& =\sum_{k=1}^{n} V_{k} \sum_{j=1}^{p}(-1)^{j+1} T_{\nu_{j}} \xi^{p}\left(k, \nu_{1}, \cdots, \hat{\nu}_{j}, \cdots, \nu_{p}\right) \\
& =\left(\sum_{k=1}^{n} V_{k} T_{k}\right) \xi^{p}\left(\nu_{1}, \cdots, \nu^{p}\right)=\xi^{p}\left(v_{1}, \cdots, \nu_{p}\right),
\end{aligned}
$$

where we have used the relation

$$
T_{k} \xi^{p}\left(\nu_{1}, \cdots, \nu_{p}\right)=\sum_{j=1}^{p}(-1)^{j+1} T_{\nu j} \xi^{p}\left(k, \nu_{1}, \cdots, \hat{\nu}_{j}, \cdots, \nu_{p}\right),
$$

obtained from $\delta_{T}^{p} \xi^{p}=0$. (In particular, we have obtained the nonsingularity of $T \mid Z$.) Consequently the norm of the map $\xi^{p} \rightarrow \eta^{p}+$ $\mathfrak{N}\left(\delta_{T \mid Z}^{p}\right)$ has the estimation

$$
\left\|\eta^{p}+\mathfrak{R}\left(\delta_{T \mid Z}^{p}\right)\right\| \leqq\left\|\eta^{p}\right\| \leqq C\left\|\xi^{p}\right\|
$$

where $C>0$ does not depend on $Z$, as it follows from (4.4). More general, in order to estimate the norm of (4.3), if $\xi=\bigoplus_{p=0}^{n} \xi^{p} \in \Re\left(\delta_{T \mid Z}\right)$ we can choose a solution $\eta=\bigoplus_{p=0}^{n} Z^{(n)}$ of the equation $\delta_{T} \eta=\xi$ such that

$$
\left\|\eta+\mathfrak{R}\left(\delta_{T \mid Z}\right)\right\| \leqq\|\eta\| \leqq C\|\xi\|,
$$

where $C>0$ does not depend on $Z$. According to the definition (4.2) we have then

$$
\kappa(T, \Re)=\kappa\left(\delta_{T}, \Re^{(n)}\right)<+\infty .
$$

and the proof is complete.

5. We shall deal in what follows with some special problems of spectral theory of commuting systems of operators on a Banach space $X$.

We recall that a spectral capacity on $C^{n}$ [3], [2] is a map assigning to every closed set $F \subset C^{n}$ a closed subspace $X(F) \subset X$, with the properties: 
(1) $X(\varnothing)=\{0\} ; X\left(C^{n}\right)=X$.

(2) $X\left(\bigcap_{k=1}^{\infty} F_{k}\right)=\bigcap_{k=1}^{\infty} X\left(F_{k}\right)$, for any sequence of closed sets $\left\{F_{k}\right\}_{k=1}^{\infty}$ in $C^{n}$

(3) $\sum_{k=1}^{p} X\left(\bar{G}_{k}\right)=X$ for any open covering $\left\{G_{k}\right\}_{k=1}^{p}$ of $\mathbb{C}^{n}$.

If the property (3) is true only for $p \leqq m$, with a fixed $m \geqq 2$, then the map $F \rightarrow X(F)$ is an m-spectral capacity [2], [7].

A commuting system of operators $T=\left(T_{1}, \cdots, T_{n}\right)$ is called $m$-decomposable (decomposable) if there exists an $m$-spectral (a spectral) capacity $F \rightarrow X(F)$ ( $F$ closed in $C^{n}$ ) with the properties:

(1) each $X(F)$ is invariant under $T$;

(2) $\delta(T ; X(F)) \subset F$, for every $F$.

It is known that each $m$-decomposable system $T$ has only one $m$ spectral capacity [7], [11].

Let $T=\left(T_{1}, \cdots, T_{n}\right)$ be an $m$-decomposable system of operators on $X$ and $F \rightarrow X(F)$ its $m$-spectral capacity.

We shall say that $F \rightarrow X(F)$ is tempered on a set $A \subset C^{n}$ if for every $z \notin \bar{A}$ we have

$$
\kappa\left(z-T, \Re_{A}\right)<+\infty,
$$

where $\Re_{A}=\{X(F) ; F \subset A, F$ closed $\}$.

We shall say that $F \rightarrow X(F)$ is *-tempered on the closed set $K \subset C^{n}$ if for every $z \notin K$ there is an open set $H \ni z, \bar{H} \cap K=\varnothing$, such that

$$
\kappa\left(z-T^{*}, \mathfrak{R}_{K, z}^{*}\right)<+\infty,
$$

where $\mathfrak{R}_{K, z}^{*}=\left\{(X / X(\bar{G}))^{*} ; \bar{G} \cap K=\varnothing, G \supset H, G\right.$ open $\} \quad$ (the $\quad$ spaces $(X / X(\bar{G}))^{*}$ are identified here with subspaces of $\left.X^{*}\right)$.

For any set $A \subset C^{n}$ we define

$$
X(A)=\bigcup\{X(F) ; F \subset A, F \text { closed }\} .
$$

Since every spectral capacity is monotone, it is clear that $X(A)$ is a subspace, not necessarily closed, of $X$.

When a spectral capacity is tempered (*-tempered) on every (closed) set, we shall call it simply tempered (*-tempered).

Proposition 5.1. Let $T=\left(T_{1}, \cdots, T_{n}\right)$ be an m-decomposable system of operators on $X$ and $F \rightarrow X(F)$ its m-spectral capacity.

(a) If $F \rightarrow X(F)$ is tempered on $A \subset C^{n}$ then $\delta(T ; \overline{X(A)}) \subset \bar{A}$.

(b) If $F \rightarrow X(F)$ is *-tempered on the closed set $K \subset C^{n}$ then

$$
\delta\left(T^{*} ;\left(X / \overline{X\left(C^{n} \backslash K\right)}\right)^{*}\right) \subset K .
$$

Proof. Fix $z \notin A$ and consider the family of closed subspaces 
$\Re_{A}$ which appears in (5.1). It is clear that $\Re_{A}$ is increasingly directed. According to (5.1) and Theorem 4.3, $z-T$ is nonsingular on c.l.m. $\{X(F)\}_{F \subset A}=\overline{X(A)}$. In this way we have (a).

In order to prove (b), first of all let us show that

$$
\delta(T ; X / X(\bar{G})) \subset C^{n} \backslash G,
$$

for any open set $G \subset C^{n}$. We shall use an argument from [11]. Let $H$ be another open set such that $G \cup H=C^{n}$. We have then $X=$ $X(\bar{G})+X(\bar{H})$, because $T$ is $m$-decomposable. Notice that $X / X(\bar{G})$ is isomorphic to $X(\bar{H}) / X(\bar{G} \cap \bar{H})$, hence

$$
\delta(T ; X / X(\bar{G}))=\delta(T ; X(\bar{H}) / X(\bar{G} \cap \bar{H})) \subset \bar{H} \cup(\bar{G} \cap \bar{H})=\bar{H},
$$

according to a Taylor's result on spectral inclusions [9, Lemma 1.2]. As $H$ is arbitrary with the property $G \cup H=C^{n}$, we obtain (5.3).

Secondly, note that if $z \notin K$ is fixed, we have

$$
\left.\left.\left(X / \overline{X\left(C^{n} \backslash K\right.}\right)\right)^{*}=\bigcap\{X / X(\bar{G}))^{*} ; \bar{G} \cap K=\varnothing, G \supset H, G \text { open }\right\},
$$

where $H$ is chosen such that the relation (5.2) is fulfild. On account of Theorem 4.4 and the relations (5.2) and (5.3) we obtain that $z-T^{*}$ is nonsingular on $\left.\left(X \overline{X\left(C^{n} \backslash K\right.}\right)\right)^{*}$.

Proposition 5.1(b) suggests the definition of a "dual capacity" by the formula

$$
\left.X^{*}(F)=\left(X / \overline{X\left(C^{n} \backslash F\right.}\right)\right)^{*},
$$

where $F \subset C^{n}$ is arbitrary, as a natural extension of the onedimensional case [6]. In order to apply Proposition 5.1, we must assume that the spectral capacity $F \rightarrow X(F)$ is $*$-tempered on each closed set. It is beyond our scope to develop here a theory of duality for spectral capacities in several dimensions. We have only illustrated some of the difficulties of such an attempt.

For $n=1$ the formulas (5.1) and (5.2) are automatically fulfiled.

Proposition 5.2. Let $T$ be an m-decomposable operator. Then its $m$-spectral capacity $F \rightarrow X(F)(F \subset C, F$ closed) is tempered and *-tempered.

Proof. Fix an arbitrary set $A \subset C$ and take $z \notin \bar{A}$. According to Proposition 1.1 we have then

$$
\sup _{\substack{F \subset A \\ F \text { closed }}}\left\|((z-T) \mid X(F))^{-1}\right\| \leqq\left\|((z-T) \mid X(\bar{A}))^{-1}\right\|,
$$

hence $F \rightarrow X(F)$ is tempered on $A$. 
Now, it is known that the formula (5.4) defines the 2-spectral capacity of $T[6]$. From the formula (5.3) we obtain

$$
\delta\left(T^{*} ;(X / X(\bar{G}))^{*}\right)=\delta(T ; X / X(\bar{G})) \subset C \backslash G,
$$

hence $(X / X(\bar{G}))^{*} \subset X^{*}(C \backslash G)$, the spaces $X^{*}(F)$ being spectra maximal (see [6] for details). If $K \cup C$ is a closed set and $z \in K$ is arbitrary, if we define

$$
H=\{w \in C ; \operatorname{dist}(w, K)\}>\frac{1}{2} \operatorname{dist}(z, K),
$$

we have for any $G \supset H, \bar{G} \cap K=\varnothing$,

$$
\begin{aligned}
\left.\|(z-T)(X / X(\bar{G}))^{*}\right)^{-1} \| & \leqq\left\|\left(\left(z-T^{*}\right) \mid X^{*}(C \backslash G)\right)^{-1}\right\| \\
& \leqq\left\|\left(\left(z-T^{*}\right) \mid X^{*}(C \backslash H)\right)^{-1}\right\|,
\end{aligned}
$$

whence $F \rightarrow X(F)$ follows *-tempered.

In several dimensions, the existence of a result similar with Proposition 5.2 is not certain; it becomes yet less certain in virtue of our examples in the fourth section. However, the answer is positive for classes of systems satisfying the conditions of Theorem 4.5. Such examples are, for instance, the classes of systems having functional calculi [4], [1]. For the sake of simplicity we shall consider only a particular case (see [1] for details).

Let $\mathscr{C}^{\infty}\left(C^{n}\right)$ be the algebra of all indefinitely differentiable complex function in $C^{n}=R^{2 n}$.

A functional calculus is a continuous homomorphism $U$ of the unital algebra $\mathscr{C}^{\infty}\left(C^{n}\right)$ into the unital algebra $\mathfrak{B}(X)$. Denote by $T_{j}=U\left(z_{j}\right)$, where $z \rightarrow z_{j}$ stand for the coordinate functions $(j=1$, $\cdots, n)$. The system $T=\left(T_{1}, \cdots, T_{n}\right)$ will be called a generalized scalar system. Such a system is decomposable and its spectral capacity is given by

$$
X(F)=\bigcap\{\Re(U(f)) ; \text { supp } f \cap F=\varnothing\},
$$

where supp $f$ denotes the support of $f$ in $C^{n}$, and $F$ is an arbitrary closed set in $C^{n}$. It is known that $T \mid X(F)$ is again a generalized scalar system, for any closed $F$.

Proposition 5.3. Let $T=\left(T_{1}, \cdots, T_{n}\right)$ be a generalized scalar system. Then its spectral capacity is tempered and *-tempered.

Proof. Let $A$ be an arbitrary set in $C^{n}$ and $z \in \bar{A}$ a fixed point, $z=\left(z_{1}, \cdots, z_{n}\right)$. Then the functions

$$
\varphi_{j}(w)=\overline{\left(z_{j}-w_{j}\right)} / \sum_{k=1}^{n}\left|z_{k}-w_{k}\right|^{2}
$$


are defined in a neighborhood of $\bar{A}$, therefore the operators $V_{j}=$ $(U \mid X(\bar{A}))\left(\varphi_{j}\right)$ are correctly defined and we have $\sum_{j=1}^{n}\left(z_{j}-T_{j}\right) V_{j}=1$ on $X(\bar{A})$. Since the spaces $X(F)$ are invariant under $T_{j}$ and $V_{j}$ for any $F \subset A, F$ closed, we may apply Theorem 4.5 and get that the spectral capacity $F \rightarrow X(F)$ is tempered on $A$.

Since the system $T^{*}=\left(T_{1}^{*}, \cdots, T_{n}^{*}\right)$ is generalized scalar on $X^{*}$, the proof that $F \rightarrow X(F)$ is $*$-tempered is similar and will be omitted.

\section{REFERENCES}

1. E. Albrecht, Funktionalkalküle in mehreren Veränderlichen für stetige lineare Operatoren auf Banachräumen, Manuscripta Math., 14 (1974), 1-40.

2. E. Albrecht and F.-H. Vasilescu, On spectral capacities, Rev. Roum. Math. Pures Appl., 19 (1974), 701-705.

3. C. Apostol, Spectral decomposition and functional calculus, Rev. Roum. Math. Pures Appl. 13 (1968), 1483-1530.

4. I. Colojoară and C. Foias, Generalized spectral operators, Gordon and Breach, New York, 1968.

5. C. Foias, Spectral capacities and decomposable operators, Rev. Roum. Math. Pures Appl. 13 (1968), 1539-1545.

6. S. Frunză, $A$ duality theorem for decomposable operators, Rev. Roum. Math. Pures Appl. 16 (1971), 1055-1058.

7. - Théorie spectrale locale en plusieurs variable, C. R. Acad. Sc. Paris, Série

A, 277 (1973), 785-787.

8. T. Kato, Perturbation Theory for Linear Operators, Springer-Verlag, Berlin-Heidelberg-New York, 1966.

9. J. L. Taylor, $A$ joint spectrum for several commuting operators, J. Functional Analysis 6 (1970), 172-191.

10. - The analytic functional calculus for several commuting operators, Acta Math., 125 (1970), 1-38.

11. F.-H. Vasilescu, An application of Taylor's functional calculus, Rev. Roum. Math. Pures Appl., 19 (1974), 1165-1167.

12. - A characterization of the joint spectrum in Hilbert spaces, Rev. Roum. Math. Pures Appl., (to appear).

Received April 18, 1975.

Institute of Mathematics

BUCHAREST, ROMANIA 


\title{
PACIFIC JOURNAL OF MATHEMATICS
}

\section{EDITORS}

RichaRd ARENS (Managing Editor)

University of California

Los Angeles, California 90024

\section{R. A. Beaumont}

University of Washington

Seattle, Washington 98105
J. DUGUNDJI

Department of Mathematics University of Southern California Los Angeles, California 90007

D. Gilbarg and J. Milgram

Stanford University

Stanford, California 94305

\section{ASSOCIATE EDITORS}
E. F. BECKENBACH
B. H. NeUmanN
F. WOLF
K. YoshIDA

\section{SUPPORTING INSTITUTIONS}

\author{
UNIVERSITY OF BRITISH COLUMBIA \\ CALIFORNIA INSTITUTE OF TECHNOLOGY \\ UNIVERSITY OF CALIFORNIA \\ MONTANA STATE UNIVERSITY \\ UNIVERSITY OF NEVADA \\ NEW MEXICO STATE UNIVERSITY \\ OREGON STATE UNIVERSITY \\ UNIVERSITY OF OREGON \\ OSAKA UNIVERSITY
}

\author{
UNIVERSITY OF SOUTHERN CALIFORNIA \\ STANFORD UNIVERSITY \\ UNIVERSITY OF TOKYO \\ UNIVERSITY OF UTAH \\ WASHINGTON STATE UNIVERSITY \\ UNIVERSITY OF WASHINGTON \\ AMERICAN MATHEMATICAL SOCIETY
}

The Supporting Institutions listed above contribute to the cost of publication of this Journal, but they are not owners or publishers and have no responsibility for its content or policies.

Mathematical papers intended for publication in the Pacific Journal of Mathematics should be in typed form or offset-reproduced, (not dittoed), double spaced with large margins. Underline Greek letters in red, German in green, and script in blue. The first paragraph or two must be capable of being used separately as a synopsis of the entire paper. Items of the bibliography should not be cited there unless absolutely necessary, in which case they must be identified by author and Journal, rather than by item number. Manuscripts, in triplicate, may be sent to any one of the editors. Please classify according to the scheme of Math. Reviews, Index to Vol. 39. All other communications should be addressed to the managing editor, or Elaine Barth, University of California, Los Angeles, California, 90024.

The Pacific Journal of Mathematics expects the author's institution to pay page charges, and reserves the right to delay publication for nonpayment of charges in case of financial emergency.

100 reprints are provided free for each article, only if page charges have been substantially paid. Additional copies may be obtained at cost in multiples of 50 .

The Pacific Journal of Mathematics is issued monthly as of January 1966. Regular subscription rate: $\$ 72.00$ a year (6 Vols., 12 issues). Special rate: $\$ 36.00$ a year to individual members of supporting institutions.

Subscriptions, orders for back numbers, and changes of address should be sent to Pacific Journal of Mathematics, 103 Highland Boulevard, Berkeley, California, 94708.

PUBLISHED BY PACIFIC JOURNAL OF MATHEMATICS, A NON-PROFIT CORPORATION

Printed at Kokusai Bunken Insatsusha (International Academic Printing Co., Ltd.), 8-8, 3-chome, Takadanobaba, Shinjuku-ku, Tokyo 160, Japan.

Copyright (C) 1975 by Pacific Journal of Mathematics Manufactured and first issued in Japan 


\section{Pacific Journal of Mathematics}

\section{Vol. 59, No. $2 \quad$ June, 1975}

Aharon Atzmon, A moment problem for positive measures on the unit disc ........

Peter W. Bates and Grant Bernard Gustafson, Green's function inequalities for

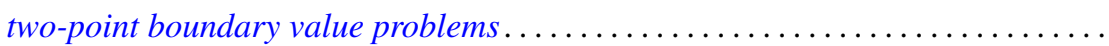

Howard Edwin Bell, Infinite subrings of infinite rings and near-rings ...........

Grahame Bennett, Victor Wayne Goodman and Charles Michael Newman, Norms of

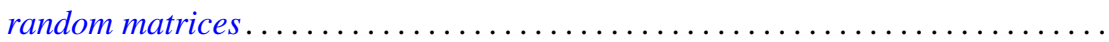

Beverly L. Brechner, Almost periodic homeomorphisms of $E^{2}$ are periodic.........

Beverly L. Brechner and R. Daniel Mauldin, Homeomorphisms of the plane ........

Jia-Arng Chao, Lusin area functions on local fields ......................

Frank Rimi DeMeyer, The Brauer group of polynomial rings ...............

M. V. Deshpande, Collectively compact sets and the ergodic theory of

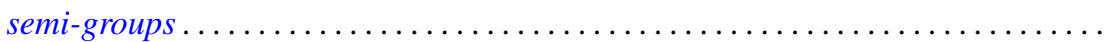

Raymond Frank Dickman and Jack Ray Porter, $\theta$-closed subsets of Hausdorff

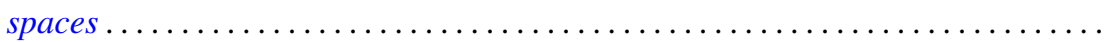

Charles P. Downey, Classification of singular integrals over a local field .......... 407

Daniel Reuven Farkas, Miscellany on Bieberbach group algebras . . . . . . . . . . . .

Peter A. Fowler, Infimum and domination principles in vector lattices . . . . . . . . . .

Barry J. Gardner, Some aspects of T-nilpotence. II: Lifting properties over

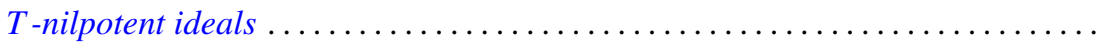

Gary Fred Gruenhage and Phillip Lee Zenor, Metrization of spaces with countable

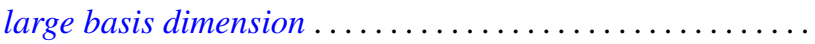

J. L. Hickman, Reducing series of ordinals...

Hugh M. Hilden, Generators for two groups related to the braid group ...

Tom (Roy Thomas Jr.) Jacob, Some matrix transformations on analytic sequence

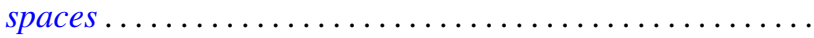

Elyahu Katz, Free products in the category of $k_{w}$-groups . .....

Tsang Hai Kuo, On conjugate Banach spaces with the Radon-Nikodým property...

Norman Eugene Liden, $K$-spaces, their antispaces and related mappings ...

Clinton M. Petty, Radon partitions in real linear spaces ........

Alan Saleski, A conditional entropy for the space of pseudo-Menger maps ....

Michael Singer, Elementary solutions of differential equations .

Eugene Spiegel and Allan Trojan, On semi-simple group algebras. I. . .

Charles Madison Stanton, Bounded analytic functions on a class of open Riemann

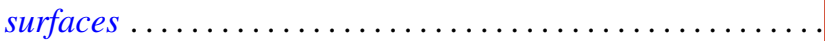

Sherman K. Stein, Transversals of Latin squares and their generalizations ....

Ivan Ernest Stux, Distribution of squarefree integers in non-linear sequences . . .

Lowell G. Sweet, On homogeneous algebras ................

Lowell G. Sweet, On doubly homogeneous algebras .......... .

Florian Vasilescu, The closed range modulus of operators ......

Arthur Anthony Yanushka, A characterization of the symplectic groups $\operatorname{PSp}(2 m, q)$

as rank 3 permutation groups... 